\title{
Identification of Safety Outcomes for Canadian Home Care Clients Evidence from the Resident Assessment Instrument - Home Care reporting system concerning emergency room visits
}

\author{
Diane M. Doran, John Hirdes, Jeff Poss, Micaela Jantzi, Régis Blais, G. Ross Baker and Jennie Pickard
}

\begin{abstract}
Problems of patient safety have been well documented in hospitals. However, we have very limited data about patient safety problems among home care clients. The purpose of this study was to assess the burden of safety problems among Canadian home care clients using data collected through the Resident Assessment Instrument - Home Care (RAI HC), and to explore the role of age and patient safety risk factors in explaining variations in adverse outcomes, with a particular focus on emergency room visits. The study methodology involved a secondary analysis of data collected through the Canadian Home Care Reporting System. The study sample consisted of all home care clients who qualified to receive an RAI HC assessment from Ontario, Nova Scotia and Winnipeg Regional Health Authority for the 2003-2007 reporting period. There were a total of 30,396 cases with a paired intake and 12-month follow-up assessment available for analysis.

New falls, unintended weight loss, new emergency room (ER) visits and new hospital visits were the most prevalent adverse outcomes. A history of falls, a cancer diagnosis, polypharmacy, receiving an anxiolytic medication and receiving an antidepressant medication were
\end{abstract}

associated with an increased risk of ER visits, while low selfreliance and limitation in activities of living were associated with a decreased risk of ER visits. Understanding clients' risk profiles is foundational to effective patient care.

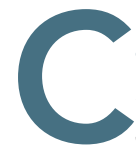

anada faces enormous healthcare challenges driven by realities that include a shortage of health professionals such as nurses, an aging workforce, issues in interprofessional care, advancing technologies, demographic changes, the introduction of electronic health records, increasing patient complexity and the need for effective chronic disease management. Underlying all of this is a recognized concern about the safety of care.

Problems of patient safety have been well documented in hospitals (Baker et al. 2004). In contrast, only limited data are available about patient safety problems among home care clients, where care differs from the hospital setting in terms of the nature of formal service provision, the role of family members and the characteristics of the individuals receiving care (Hirdes et al. 2004). Two recent studies, one conducted in the United States (Madigan and Fortinsky 1999) and the other in Ontario (Sears 2008), reported that $13 \%$ of home care clients experienced an adverse event. Clients who experienced such events were gener- 
ally older, had more depressive symptoms and behavioural problems, were more functionally impaired (Madigan 2007), had Parkinson's disease, received psychotropic medications or were left alone for short or long periods of time (Sears 2008).

A recent Canadian study provided valuable evidence on the nature and prevalence of patient safety risks and outcomes among Canadian home care clients (Doran et al. in press). Using Resident Assessment Instrument - Home Care (RAI HC) (Hirdes et al. 1999; Morris et al. 1997) data for 239,011 Canadian home care clients from Nova Scotia, Ontario and the Winnipeg Regional Health Authority, it was determined that a new fall, unintended weight loss, a new visit to the emergency room (ER) and a new hospitalization were the most frequent adverse outcomes among Canadian home care clients. Other adverse outcomes identified were new cognitive decline, a new urinary tract infection, deterioration in or a new pressure ulcer and new pneumonia. Significant variations in adverse outcomes were also found between regions of the country. This finding provided the impetus for the second study reported in this paper. The present paper aims to explore the extent to which age explains regional differences in adverse outcomes among Canadian home care clients and to explore the extent to which safety risk factors account for variations in regional rates of adverse outcome, after controlling for age. To do so, the focus is on one particular adverse outcome: ER visits.

ER visits are of interest for two reasons: first, a visit to the ER was among the most prevalent adverse outcomes for home care clients in our study; and, second, the Ontario Ministry of Health and Long-Term Care has recently invested \$109 million to reduced wait times in ERs (Ministry of Health and Long-Term Care 2008a, 2008b). If it is possible to identify factors that increase the risk of ER visits and to intervene to reduce that risk, it may be feasible to reduce wait times by preventing unnecessary ER use by home care clients.

\section{Theoretical Framework}

The conceptualization of patient safety risk factors and adverse outcomes was guided by the World Health Organization (WHO) conceptual framework for the International Classification for Patient Safety (WHO 2008). The WHO framework provides a way to conceptualize the interrelationship among the factors that contribute to patient safety incidents and their consequences. WHO defines a contributing factor or hazard as a circumstance, action or influence that is thought to have played a part in the origin or development of a patient safety incident. A patient safety incident is defined as "an event or circumstance which could have resulted, or did result, in unnecessary harm to a patient" (WHO 2008: 16). Patient safety incidents are classified within the framework into 15 different types of process errors. Examples include errors related to handover, diagnosis, missing documentation, prescribing and administration. A patient outcome is defined as "the impact upon a patient which is wholly or partially attributable to an incident" (WHO 2008: 17). The WHO framework also identifies organizational outcomes associated with patient safety incidents, which are defined as "the impact upon an organization which is wholly or partially attributable to an incident. Examples would be adverse publicity and additional use of resources" (WHO 2008: 9).

\section{The risk factors that were found to predict the occurrence of ER visits ... can provide the basis for targeted interventions to reduce the risk of ER visits among home care clients.}

Safety is defined by WHO as "freedom from hazard," and hazard is defined as a "circumstance, agent or action which can lead to or increase risk" (WHO 2008: 6). Patient safety is defined as "freedom for patient from unnecessary harm or potential harm associated with healthcare" (WHO 2008: 7). WHO considers healthcare-associated harm as "harm arising from or associated with plans or actions taken during the provision of health care rather than an underlying disease or injury" (2008: 7). The WHO framework is useful for studying safety in the home care context because it recognizes that healthcare is not limited to medical care provided by health professionals, and it includes self-care. This recognition is consistent with the context of home care, where much of the care is provided by informal caregivers, such as family members.

\section{Study Purpose}

The purpose of this study was to explore the role of age and patient safety risk factors in explaining variations in adverse outcomes for Canadian home care clients, with a particular focus on ER visits, using data collected through the RAI HC assessment instrument.

The specific objectives of this study were as follows:

- To explore the extent to which age explains regional variations in adverse outcomes among Canadian home care clients

- Utilizing one adverse outcome, ER visits, to explore the extent to which patient safety risk factors explain regional variations in ER visits among Canadian home care clients

\section{Methodology}

The study methodology involved a secondary analysis of RAI $\mathrm{HC}$ data available through the Canadian Institute for Health Information (CIHI) Home Care Reporting System. The RAI $\mathrm{HC}$ assessment provides a comprehensive profile of Canadian 
Table 1. Patient safety risk factors identified through the RAI HC assessment tool

\begin{tabular}{|c|c|}
\hline & Safety Risk Factor \\
\hline \multirow[t]{7}{*}{ Client characteristic } & Decline in physical function \\
\hline & Decline in mental function \\
\hline & Decline in cognitive function \\
\hline & Hearing deficit \\
\hline & Vision deficit \\
\hline & Social isolation with distress \\
\hline & HIV or tuberculosis infection \pm others in the home \\
\hline \multirow{6}{*}{$\begin{array}{l}\text { Client behavioural } \\
\text { characteristic }\end{array}$} & History of two or more falls \\
\hline & Non-adherence to medication \\
\hline & Substance abuse \\
\hline & $\begin{array}{l}\text { Smoking } \pm \text { oxygen in the home; } \pm \text { others in the } \\
\text { home (exposure to second-hand smoke) }\end{array}$ \\
\hline & Aggressive behaviour \\
\hline & $\begin{array}{l}\text { Morbid obesity and requiring weight-bearing } \\
\text { assistance for transfer }\end{array}$ \\
\hline \multirow[t]{3}{*}{ Client living situation } & Lives alone and decline in physical function \\
\hline & Lives alone and decline in cognition \\
\hline & Unsafe housing \\
\hline \multirow{2}{*}{$\begin{array}{l}\text { Healthcare management } \\
\text { factors }\end{array}$} & Polypharmacy \pm history of cognitive impairment \\
\hline & $\begin{array}{l}\text { No medication review }- \text { for clients with } \\
\text { polypharmacy } \pm \text { history of cognitive impairment }\end{array}$ \\
\hline
\end{tabular}

HIV = human immunodeficiency virus; RAI HC = Resident Assessment Instrument - Home Care

available from Winnipeg Regional Health Authority, Ontario and Nova Scotia because these three jurisdictions have agreements that permit their home care reporting data to be shared for research purposes and because there are sufficiently large numbers of cases to enable the risk analysis.

\section{Sample}

The study sample consisted of all home care clients who qualified to receive an RAI HC assessment from three provinces (Ontario, Nova Scotia and Manitoba [Winnipeg Regional Health Authority]). Only clients who are expected to receive services for 60 days or longer receive the RAI HC assessment. Therefore, short-stay clients were not included in the study sample. The study sample was created using RAI HC data from the three provinces for the 2003-2007 reporting period. The adverse outcome analysis used pairs of the intake assessment and the subsequent assessment in order to determine the incidence of an adverse outcome. Because the time interval between the intake assessment and subsequent assessment could impact the occurrence of an adverse outcome, the sample was limited to home care clients who had received a 12-month reassessment plus or minus 60 days. There were a total of 30,396 cases available for analysis: 26,156 from Ontario, 3,651 from Nova Scotia and 589 from Winnipeg Regional Health Authority.

\section{Study Variables and Definitions}

The WHO framework guided the conceptualization of the patient safety variables in our study. Two types of safety indicators were studied: safety risk/hazards and adverse outcomes. These two indicators are related but conceptually distinct. Safety risks/hazards are

home care clients, their environment, services and outcomes (Canadian Home Care Association 2008). It includes a series of outcome measures such as cognitive performance, activities of daily living (ADL) scales, an index of social engagement, a depression rating scale, falls and pressure ulcers. Agreement was obtained from CIHI for access to the data. Personal identifiers, such as names and health card numbers, were stripped from the record to prevent identification prior to the transmission of the data to the research group. The study received ethical approval from the University of Toronto Research Ethics Review Board. This project focused on analyzing the RAI HC data currently defined as "characteristics of the client or the living situation that place a client at risk for adverse outcome" (Doran et al. 2009). The safety risk variables were based on evidence from the literature (Baker et al. 2004; Madigan 2007; Masotti et al. 2007; Sears 2008) and were operationally defined in a previous article (Doran et al. 2009). They were categorized into four types: client physical or cognitive factors, behavioural factors, living situation and healthcare management factors. Examples of patient safety risk factors for each category are provided in Table 1. An adverse event was defined as an unintended injury or complication that resulted in disability, death, or increased use 
Table 2. Study sample characteristics by region

\begin{tabular}{|c|c|c|c|c|c|c|c|}
\hline & & \multicolumn{2}{|c|}{ Nova Scotia } & \multicolumn{2}{|c|}{ Ontario } & \multicolumn{2}{|c|}{ Winnipeg } \\
\hline & & $n=3,651$ & $\%$ & $n=26,156$ & $\%$ & $n=589$ & $\%$ \\
\hline \multirow[t]{4}{*}{ Age categories (yr) } & $<65$ & 506 & 13.9 & 3,450 & 13.2 & 77 & 13.1 \\
\hline & $65-74$ & 658 & 18.0 & 4,201 & 16.1 & 80 & 13.6 \\
\hline & $75-84$ & 1,430 & 39.2 & 11,155 & 42.7 & 241 & 40.9 \\
\hline & $85+$ & 1,057 & 29.0 & 7,350 & 28.1 & 191 & 32.4 \\
\hline Female & & 2,583 & 70.5 & 17,942 & 68.5 & 399 & 67.6 \\
\hline Living alone & & 1,652 & 45.1 & 9,333 & 35.6 & 331 & 56.1 \\
\hline $\begin{array}{l}\text { Cognitive Performance Scale } \\
\text { score } \geq 2\end{array}$ & & 1,039 & 28.4 & 8,336 & 31.8 & 163 & 27.6 \\
\hline $\begin{array}{l}\text { Activities of Daily Living } \\
\text { Hierarchy score } \geq 2\end{array}$ & & 853 & 23.3 & 4,990 & 19.1 & 90 & 15.3 \\
\hline Depression Rating Score $\geq 2$ & & 270 & 7.4 & 3,583 & 13.7 & 53 & 9.0 \\
\hline CHESS $*$ score $\geq 2$ & & 1,310 & 35.8 & 10,530 & 40.2 & 206 & 34.9 \\
\hline
\end{tabular}

*The interRAI Changes in Health, End-stage disease and Signs and Symptoms (CHESS) score is a composite measure of change in health status, end-stage disease and symptoms and signs (e.g., vomiting, dehydration, weight loss and shortness of breath) and has been shown to be a strong predictor of mortality (Hirdes et al. 2003).

of healthcare resources and was caused by healthcare management (Baker et al. 2004).

\section{Analytical Strategy}

Descriptive statistics were conducted to describe the population characteristics based on gender, age, living arrangement and intake RAI HC assessment data. Incidence rates were calculated for each adverse outcome by region. Age-adjusted rates were calculated using Ontario as the population standard. Logistic regression analysis was used to determine the influence of age and patient safety risk factors in explaining regional variations in adverse outcomes.

\section{Results}

The results presented in Table 2 describe the study sample. The majority of clients were in the age range of $75+$ years, female, living with someone else and cognitively intact. Chi-square tests indicated significant differences in population characteristics between the three provinces. For example, there were significant differences across the three regions of the country in the proportion of clients with decline in ADL $(p<.0001)$, possible depression $(p<.0001)$ and living alone $(p<.0001)$. Although the variation was smaller, there were also significant differences in the age $(p<.0003)$ and gender $(p<.04)$ distributions of clients across the three provinces.

The age-adjusted rates for potential adverse outcomes among Canadian home care clients are presented in Table 3. These were identified as potential adverse outcomes to be consistent with the WHO framework, which links adverse outcomes to a process error (i.e., safety incident). Further work would be required to make such a linkage. A new fall, unintended weight loss, a new ER visit and a new hospital visit were the most frequently occurring potential adverse outcomes.

\section{Relationship between Safety Risks and ER Visits}

One adverse outcome, ER visits, was selected in order to explore the role of risk factors in explaining regional variations in adverse outcomes. Three logistic regression models were run. The first model included a variable representing region, with Winnipeg Regional Health Authority as the reference variable. The second model included a variable representing age categories, and the third model included the risk factors identified in a previous article (Doran et al. 2009) as well as potential risk factors identified in other practice settings such as long-term care (Hirdes et al. 2003). Table 4 provides a summary of the results of the logistic regression analyses. 
Table 3. Twelve-month age-adjusted rates for potential adverse outcomes among Canadian home care clients

\begin{tabular}{|c|c|c|c|c|}
\hline & Ontario $(n=26,156)$ & Nova Scotia $(n=3,651)$ & Winnipeg $(n=589)$ & Overall $(N=30,396)$ \\
\hline Potential Adverse Outcome & $\begin{array}{c}\text { Frequency } \\
\text { (Age-Adjusted } \\
\text { Weighted \%) }\end{array}$ & $\begin{array}{c}\text { Frequency } \\
\text { (Age-Adjusted } \\
\text { Weighted \%) }\end{array}$ & $\begin{array}{c}\text { Frequency } \\
\text { (Age-Adjusted } \\
\text { Weighted \%) }\end{array}$ & $\begin{array}{l}\text { Age-Adjusted } \\
\text { Weighted \% }\end{array}$ \\
\hline New fall & $2,866(10.9)$ & $417(11.4)$ & $59(9.9)$ & 11.0 \\
\hline Unintended weight loss & $2,433(9.3)$ & $319(8.8)$ & $42(7.3)$ & 9.2 \\
\hline New ER visit & $2,317(8.9)$ & 290 (7.9) & $22(3.6)$ & 8.7 \\
\hline New hospital visit & $1,709(6.5)$ & $343(9.4)$ & $19(3.2)$ & 6.8 \\
\hline Cognitive performance decline & $1,591(6.1)$ & $390(10.7)$ & $32(5.4)$ & 6.6 \\
\hline New urinary tract infection & $580(2.2)$ & $104(2.8)$ & $9(1.6)$ & 2.3 \\
\hline Pressure ulcer deterioration & $500(1.9)$ & $63(1.7)$ & $7(1.1)$ & 1.9 \\
\hline New pressure ulcer & 464 (1.8) & $60(1.7)$ & $7(1.1)$ & 1.7 \\
\hline New pneumonia & $270(1.0)$ & $63(1.7)$ & $7(1.2)$ & 1.1 \\
\hline New bowel problem, e.g., constipation & $184(0.7)$ & $40(1.1)$ & $2(0.4)$ & 0.7 \\
\hline New dehydration & $148(0.6)$ & $21(0.6)$ & $2(0.3)$ & 0.6 \\
\hline New caregiver decline & 746 (2.8) & 217 (5.9) & $15(2.4)$ & 3.2 \\
\hline
\end{tabular}

The results of the first logistic regression model presented in Table 4 indicate that Nova Scotia and Ontario home care clients had higher odds ratios for ER visits than did clients in the Winnipeg Regional Health Authority. The results of the second logistic regression model indicate that clients less than 65 years of age had slightly higher odds of ER visits than did older clients, and that the regional differences are still significant. The small change in the c-statistic (.52 versus .51) for model two indicates that the age variable contributes very little to improve the model fit. The third model, which included the other risk factors, produced an improved model fit as evidenced by a c-statistic of .57 , although the regional differences were still significant. The factors that increased risk of ER visits by home care clients were a history of two or more falls, polypharmacy (i.e., five or more medications), a cancer diagnosis, receiving an anxiolytic medication and receiving an antidepressant medication. Decreased ADL and lower self-reliance decreased the risk of ER visits.

\section{Discussion \\ Limitations}

It was not possible to link the occurrence of a potential adverse outcome identified through the RAI HC reporting system to a patient safety incident (i.e., process error) as conceptualized in the WHO (2008) safety framework. There are several steps that would need to be pursued in order to make such a linkage. First, it would be necessary to conduct a chart audit for the purpose of identifying the underlying patient safety incident that contributed to an adverse outcome. This would enable the identification of adverse outcomes that were preventable and were attributable to healthcare management. Second, it would be helpful to link the cases identified through the RAI HC reporting system to the hospital discharge administrative database to identify reasons for re-hospitalization or reasons for visits to the ER. This would enable a determination of whether the return visit to the hospital or ER was associated with home healthcare management. A second limitation is that not all home care clients are represented in the database because only long-stay clients qualify for an RAI $\mathrm{HC}$ assessment. Thus, the study findings are only generalizable to long-stay clients. This may change over time as provinces and territories move toward the use of the interRAI Contact Assessment as the screening methodology for home care intake. That instrument will be used to support decision-making related to the need for RAI HC assessments in all home care intakes, not just for long-stay clients. 
Table 4. Logistic regression models

\begin{tabular}{|c|c|c|c|c|c|c|c|}
\hline & & \multicolumn{2}{|c|}{ Region Only } & \multicolumn{2}{|c|}{ Region and Age } & \multicolumn{2}{|c|}{ Region, Age and Other Variables } \\
\hline & & $\mathbf{O R}$ & CLs & $\mathbf{O R}$ & CLs & OR & CLs \\
\hline \multirow[t]{3}{*}{ Region } & NS & 2.22 & $1.43,3.45$ & 2.21 & $1.42,3.45$ & 2.16 & $1.39,3.37$ \\
\hline & ON & 2.51 & $1.64,3.85$ & 2.50 & $1.63,3.84$ & 2.44 & $1.59,3.74$ \\
\hline & WRHA & 1.00 & Reference & 1.00 & Reference & 1.00 & Reference \\
\hline \multirow[t]{4}{*}{ Age categories (yr) } & $<65$ & & & 1.16 & $1.02,1.32$ & 1.09 & $0.96,1.24$ \\
\hline & $65-74$ & & & 1.05 & $0.93,1.19$ & 0.99 & $0.88,1.13$ \\
\hline & $75-84$ & & & 0.96 & $0.87,1.06$ & 0.93 & $0.84,1.02$ \\
\hline & $85+$ & & & 1.00 & Reference & 1.00 & Reference \\
\hline \multicolumn{8}{|l|}{ Risk factors } \\
\hline \multicolumn{8}{|l|}{ Patient characteristics } \\
\hline Two or more falls & & & & & & 1.20 & $1.08,1.33$ \\
\hline Activities of daily living & & & & & & 0.84 & $0.78,0.92$ \\
\hline Cancer diagnosis & & & & & & 1.16 & $1.03,1.32$ \\
\hline \multicolumn{8}{|l|}{ Self-reliance index } \\
\hline Healthcare management & & & & & & 0.88 & $0.80,0.98$ \\
\hline Received anxiolytic medication & & & & & & 1.17 & $1.05,1.30$ \\
\hline Received antidepressant medication & & & & & & 1.14 & $1.04,1.26$ \\
\hline Polypharmacy & & & & & & 1.50 & $1.34,1.69$ \\
\hline c-statistic & & \multicolumn{2}{|c|}{.512} & \multicolumn{2}{|c|}{.524} & \multicolumn{2}{|c|}{.570} \\
\hline
\end{tabular}

$\mathrm{CL}=$ confidence limit; $\mathrm{OR}=$ odds ratio

\section{Study Findings}

One of the recommendations from a recent symposium on adverse events in community care was the need for improved understanding of the variables associated with the occurrences of adverse events, including assessing patient risk (Masotti et al. 2009). This study helps to advance such understanding. Contrary to previous research (Baker et al. 2004), the present study findings suggest that age was not particularly helpful for explaining regional variations in adverse outcomes among longstay home care clients. For example, there were minimal differences between the age-adjusted rates for adverse outcomes and the unadjusted rates previously reported by our team (Doran et al. in press). Second, age contributed little to improving the model fit in the logistic regression analysis. This finding is not completely unexpected because there were only small differences in the age distribution of home care clients across the three Canadian provinces. Further, less than $14 \%$ of the sample was under the age of 65 years. Therefore, the age distribution of home care clients in our study sample was relatively homogeneous. The effect of this homogeneity would be to attenuate the correlation between age and adverse outcomes. In addition, age is unlikely to be a proxy for medical complexity or severity of disability in home care settings because eligibility criteria would take those characteristics into account. In other words, younger individuals 
with no real functional or cognitive impairment are unlikely to be recipients of long-term home care services. That said, it would be inappropriate to rule out age as a predictor of adverse outcome under wider age distributions where eligibility criteria reflecting age-related functional impairments are not used.

This study explored the extent to which the safety risk factors identified through the literature and in our previous work were associated with the adverse outcome of ER visits. The risk factors that were found to predict the occurrence of ER visits were a history of two or more falls, polypharmacy, a cancer diagnosis, self-reliance index, receiving an anxiolytic medication, receiving an antidepressant medication and a decline in ADL. Such predictions can provide the basis for targeted interventions to reduce the risk of ER visits among home care clients. For example, a history of two or more falls is a predictor of a future fall (Graafmans et al. 1996; Sears 2008). There is now a strong evidence base on which to intervene to prevent falls (Registered Nurses' Association of Ontario 2005). Therefore, by using the RAI HC tool to conduct a comprehensive assessment of home care clients, we should be able to identify clients at risk of falling and intervene to reduce adverse outcomes associated with falls.

\section{Regional differences in adverse outcomes persisted even after accounting for client age and baseline risk factors.}

The incidence of potential drug-drug interactions increases with increased drug use, and these interactions have been associated with ER visits and hospitalizations in previous research (Delafuente 2003; Hanlon et al. 1997). Further, drug interactions have been shown to cause a decline in functional abilities in older people (Delafuente 2003), compounding the risk of adverse outcomes such as falls. The present study finding, demonstrating a relationship between polypharmacy and ER visits, is consistent with this past research. Prudent use of medications and vigilant drug monitoring are essential to avoid adverse outcomes among elderly home care clients.

Receiving an anxiolytic medication or an antidepressant medication was associated with ER use among home care clients in this present study. These findings are also consistent with previous research by Sears (2008) and Madigan (2007), who found that receiving a psychotropic medication was associated with adverse outcomes among home care clients. Depression is a common comorbid condition for patients with chronic diseases such as chronic obstructive pulmonary disease and has been associated with increased physician visits, ER visits and hospitalizations (Coultas et al. 2007; Landi et al. 2004).

A decline in the ADL and lower self-reliance were both associated with lower odds of ER use in our study. The selfreliance index indicates independence in three or more of the following: hours of physical activity, meal preparation, ordinary housework, transportation, personal hygiene and bathing. These results are somewhat counterintuitive. ADL decline is an indicator of frailty and is typically associated with an increased need for assistance with personal care and homemaking. A systematic review of home-based nursing health promotion for older people found that preventive home visits were most effective for individuals who were not limited in basic ADL (MarkleReid et al. 2006). The authors of that review suggested one explanation of their findings was that a preventive intervention may work best at early and reversible stages in the continuum of health to disability, and that more disabled and frail clients require a more intensive intervention such as institutional care or more intensive systematic follow-up and coordination as well as more frequent intervention. Another systematic review of home-based support for older people found that frail older people who were at risk of adverse outcomes and received home support showed a significant reduction in mortality (Elkan et al. 2001). One possible explanation for the present study findings is that the home care clients with ADL decline and low selfreliance could have had high levels of home healthcare support already and may not have needed to use the ER for non-urgent problems. When the need for intensive service arises, they may be admitted directly to hospital or long-term care.

Regional differences in adverse outcomes persisted even after accounting for client age and baseline risk factors. This finding suggests that there could be other population differences among home care clients that predispose a person to an adverse outcome that were not measured in this study. Alternatively, differences in service provision across the three provinces could explain variation in adverse outcomes. This latter explanation warrants further investigation because our study was not able to fully explore the underlying healthcare processes that contributed to adverse outcomes among home care clients. Such exploration could provide valuable information about approaches to minimize risk in the future.

\section{Theoretical Implications}

The WHO (2008) conceptual framework for the International Classification for Patient Safety provided a way to conceptualize the inter-relationships among the factors that contribute to patient safety in the home care context. The interRAI HC assessment tool is particularly helpful for identifying safety hazards and clients safety outcomes, as conceptualized by WHO (2008). Consistent with the WHO framework, hazards are identified at both the client level (e.g., risk behaviour) and service level (e.g., healthcare service delivery). The WHO framework also identifies outcomes at the client/patient and organizational levels. At the client level, the interRAI HC assessment provides useful data about potential adverse outcomes. However, the interRAI HC tool is not designed to assess organizational outcomes. Other 
databases, such as the hospital discharge administrative database and the Home Care Database in Ontario, would be required to assess organizational outcomes. By linking the RAI home care reporting system with these other databases, we can begin to build a comprehensive patient safety monitoring system in Canada.

\section{Implications for Policy and Management}

The findings from this study have implications for home care managers and health policy makers. First, many of the safety risks are modifiable but require client behaviour change, health provider behaviour change and health system policy change. At a health system or healthcare organization level, policies could be developed to support best practices related to risk mitigation. An understanding of the risk factors that contribute to variation in safety outcomes should also enable health policy makers to make informed decisions about service priorities at the regional level.

\section{Conclusions}

The purpose of this study was to explore the role of age and patient safety risk factors in explaining variations in adverse outcomes for Canadian home care clients, with a particular focus on ER visits, using data collected through the RAI HC assessment instrument. A list of patient safety risks factors was developed based on the previous literature. Age differences in home care clients accounted for only a small amount of the variations in adverse outcome rates. Using one adverse outcome as an example, we demonstrated that characteristics related to the client (e.g., ADL decline, self-reliance, cancer diagnosis, history of falls) and healthcare services (polypharmacy, receiving an anxiolytic medication, receiving an antidepressant medication) were associated with ER use among home care clients. Many of these risk factors are amenable to change, which suggests that some of the ER use among home care clients could be prevented with effective risk mitigation. Further research is needed to fully explore how to effectively intervene with frail home care clients to promote client safety. HQ

\section{Acknowledgements}

We gratefully acknowledge the Canadian Patient Safety Institute
(CPSI) for funding this research. We also acknowledge the contribution of Nancy White, manager, home and continuing care, Canadian Institute of Health Information. The opinions, results and conclusions are those of the authors. No endorsement by the funding agency is intended or should be inferred.

\section{References}

Baker, G.R., P.G. Norton, V. Flintoft, R. Blais, A. Brown, J. Cox, E. Etchells, W.A. Ghalo, P. Herbert, S.R. Majumdar, M. O’Beirne, L. Pallacios-Derflingher, R.J. Reid, S. Sheps and R. Tamblyn. 2004. "The Canadian Adverse Events Study: The Incidence of Adverse Events among Hospital Patients in Canada." Canadian Medical Association Journal 170: $1678-86$.

Canadian Home Care Association. 2008. Portraits of Home Care in Canada. Ottawa, ON: Author.

Coultas D.B., D.W. Edwards, B. Barnett and P. Wludyka. 2007. "Predictors of depressive symptoms in patients with COPD and health impact." Journal of Chronic Obstructive Pulmonary Disease. 4: 23-8. 
Delafuente, J.C. 2003. "Understanding and Preventing Drug Interactions in Elderly Patients." Critical Review in Oncology/ Hematology 48: 133-43.

Doran, D.M., J. Hirdes, R. Blais, G.R. Baker, N. White, J. Pickard and M. Jantzi. 2009. "The Nature of Safety Problems among Canadian Home Care Clients: Evidence from the RAI-HC Reporting System." Journal of Nursing Management 17: 165-74.

Elkan, R., D. Kendrick, M. Dewey, M. Hewitt, J. Robinson, M. Blaor, D. Williams and K. Brummell. 2001. "Effectiveness of Home Based Support for Older People: Systematic Review and Meta-Analysis." BMJ 323: 1-9.

Graafmans, W.C., M.E. Ooms, H.M.A. Hofstee, P.D. Bezemer, L.M. Bouter and P. Lips. 1996. "Falls in the Elderly: A Prospective Study of Risk Factors and Risk Profiles." American Journal of Epidemiology 143: 1129-36.

Hanlon, J.T., K.E. Schmader, M.J. Koronkowski, M. Weinberger, P.B. Landsman, G.P. Samsa and I.K. Lewis. 1997. "Adverse Drug Events in High Risk Older Outpatients." Journal of the American Geriatrics Society 45: 945-48.

Hirdes, J.P., B.E. Fries, J.N. Morris, K. Steel, V. Mor, D. Frijters, P. Johnsson, S. LaBine, C. Schalm, M.J. Stone, G. Tear and T. Smith. 1999. "Integrated Health Information Systems Based on the RAI/ MDS Series of Assessment Instruments." Healthcare Management Forum 12(4): 30-40.

Hirdes, J.P., D.H. Frijters and G.F. Teare. 2003. "The MDS-CHESS Scale: A New Measure to Predict Mortality in Institutionalized Older People." Journal of the American Geriatrics Society 51: 96-100.

Hirdes, J., B.E. Fries, J.N. Morris, N. Ikegami, D. Zimmerman, D.M. Dalby, P. Aliaga, S. Hammer and R. Jones. 2004. "Home Care Quality Indicators (HCQIs) Based on the MDS-HC. Gerontologist 44: 665-79.

Johnson, K.G. 2006. "Adverse Events among Winnipeg Home Care Clients." Healthcare Quarterly 9: 127-34.

Landi, F., G. Onder, M. Cesari, C. Barillaro, F. Lattanzio, P.U. Carbonin and R. Bernabei. 2004. "Comorbidity and Social Factors Predicted Hospitalization in Frail Elderly Patients." Journal of Clinical Epidemiology 57: 832-36.

Madigan, E.A. 2007. "A Description of Adverse Events in Home Healthcare." Home Healthcare Nurse 25: 191-97.

Madigan, E.A. and R.H. Fortinsky. 1999. "Alternative Measures of Resource Consumption in Home Care Episodes." Public Health Nursing 16: 198-204.

Markle-Reid, M., G. Browne, R. Weir, A. Gafni, J. Roberts and S.R Henderson. 2006. "The Effectiveness and Efficiency of Home-Based Nursing Health Promotion for Older People: A Review of the Literature." Medical Care Research and Review 63: 531-69.

Massotti, P., M. Green and M.A. McColl. 2009. "Adverse Events in Community Care: Implications for Practice, Policy and Research." Healthcare Quarterly 12: 69-76.

Masotti, P., M. Green, S. Shortt, D. Hunter and K. Szala-Meneok. 2007. "Adverse Events in Community Care: Developing a Research Agenda." Healthcare Quarterly 10: 63-69.

Ministry of Health and Long-Term Care. 2008a. Ontario's \$109 Million Investment to Reduce Wait Times in the Emergency Room. Toronto, ON: Author. Retrieved December 21, 2008. <http://www.health.gov.on.ca/ english/media/news_releases/archives/nr_08/may/er_alc_strategy_ combined_bg_04_20080529.pdf>.

Ministry of Health and Long-Term Care. 2008b. Renewing the Ontario Home Care Administrative System (OHCAS). Toronto, ON: Author.
Retrieved December 2008. <http://www.health.gov.on.ca/transformation/providers/information/data_initiatives/bg_ohcas.pdf>.

Morris, J.N., B.E. Fries, K. Steel, N. Igegami, R. Bernabei, G.I. Carpenter, R. Giligen, J.P. Hirdes and E. TopinKova. 1997. "Comprehensive Clinical Assessment in Community Setting: Applicability of the MDS-HC." Journal of the American Geriatrics Society 45: 1017-24.

Registered Nurses' Association of Ontario. 2005. Prevention of Falls and Fall Injuries in the Older Adult. Toronto, ON: Author.

Sears, N.A. 2008. Harm from Home Care: A Patient Safety Study Examining Adverse Events in Home Care. Toronto, ON: Department of Health Policy, Management and Evaluation, University of Toronto.

World Health Organization. 2008. The Conceptual Framework for the International Classification for Patient Safety, Version 1.0, 2007-2008. Geneva, Switzerland: Author. Retrieved December 2008. <http://www. who.int/patientsafety/taxonomy/en/>.

\section{About the Authors}

Diane M. Doran, RN, PhD, FCAHS, is Lawrence S. Bloomberg professor in patient safety at the Lawrence S. Bloomberg Faculty of Nursing, University of Toronto, Toronto, Ontario. She can be reached at diane.doran@utoronto.ca.

John Hirdes, $\mathrm{PhD}$, is a professor in the Department of Health Studies and Gerontology, Faculty of Applied Health Sciences, University of Waterloo, Waterloo, Ontario, and scientific director of the Homewood Research Institute, Guelph, Ontario.

Jeff Poss, PhD, is an assistant professor in the Faculty of Applied Health Sciences, University of Waterloo.

Micaela Jantzi, BSc, is a master's degree student in the Faculty of Applied Health Sciences, University of Waterloo.

Régis Blais, $\mathrm{PhD}$, is a professor in the Département d'administration de la santé, Université de Montréal, Montréal, Québec.

G. Ross Baker, PhD, is a professor of health policy, management, and evaluation at the University of Toronto.

Jennie Pickard, RN, MScN, is senior director of performance management and accountability at the Central East Community Care Access Centre, Whitby, Ontario. 